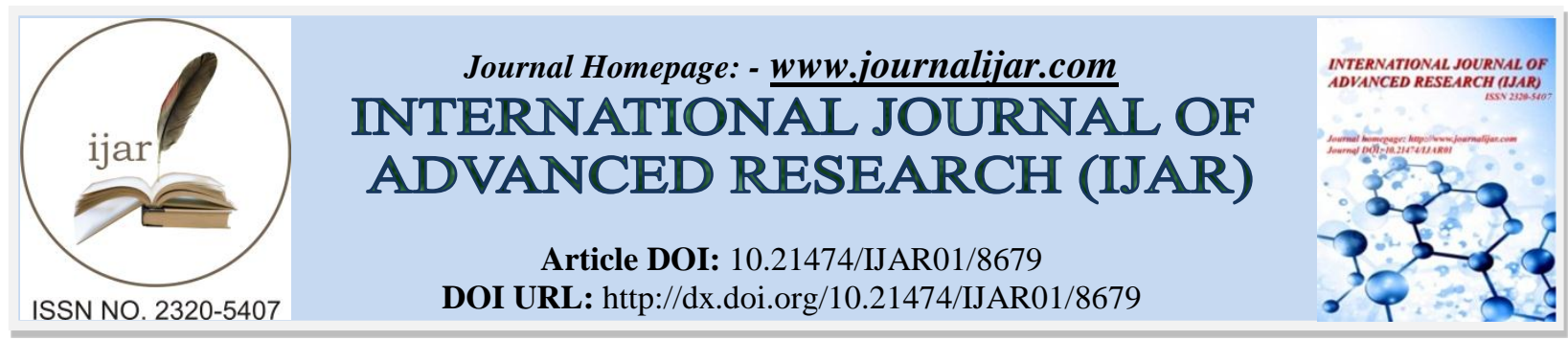

RESEARCH ARTICLE

\title{
IDIOPATHIC MEDIASTINAL LIPOMATOSIS PRESENTING AS PULMONARY EMBOLISM: A RARE CASE REPORT.
}

Hajar Hamri, Omar El Aoufir, Fatima Zahrae Laamrani and Laila Jroundi. Imaging Department, Ibn Sina Hospital, Rabat, Morocco.

\section{Manuscript Info}

\section{Manuscript History}

Received: 12 January 2019

Final Accepted: 14 February 2019

Published: March 2019

Key words:

lipomatosis - mediastinum- CT.

\begin{abstract}
Mediastinal lipomatosis is a benign condition characterized by deposition of abnormal amount of mature adipose tissue within the mediastinum. Usually asymptomatic and its incidence is underestimated. We report a symptomatic case of mediastinal lipomatosis who presented radiologically as mediastinal widening with left lower lobe segmental lung collapse.
\end{abstract}

Copy Right, IJAR, 2019. All rights reserved.

\section{Introduction:-}

Mediastinal lipomatosis is a benign condition characterized by deposition of abnormal amount of mature adipose tissue within the mediastinum. Usually asymptomatic and its incidence is underestimated. Case reports associate mediastinal lipomatosis with factors such as obesity, diabetes, Cushing's syndrome, steroid use, while other cases remain idiopathic $[1,2]$. We report a symptomatic case of mediastinal lipomatosis who presented radiologically as mediastinal widening with left lower lobe segmental lung collapse.

\section{Case report:}

A 46-year-old male with poorly monitored high blood pressure and no history of steroid use, diabetes, Cushing syndrome or obesity. He presented acute chest pain with dorsal irradiation. The clinical examination was unremarkable outside the blood pressure at $174 / 82 \mathrm{mmHg}$.

Chest radiograph showed mediastinal widening and opacity in left lower zone, suggestive of left lower lobe segmental lung collapse. The electrocardiogram was normal.

Chest CT angiography was requested to rule out pulmonary embolism, aneurysm of great vessels or aortic dissection. The chest CT scan showed an unencapsulated homogenous fat attenuating region extending from the upper mediastinum to the diaphragm and partially involving the left oblique fissure, which simulated segmental lung collapse on the chest X-ray (Fig.1). There was no mass effect on adjacent structures nor pleural or pericardial effusion. The mediastinal vessels were normal, with no proximal or distal pulmonary embolism.

This adipose tissue deposition was diagnosed as mediastinal lipomatosis. In the absence of any other identifiable cause, it was thought that mediastinal lipomatosis was a possible cause of the symptomatology of our patient. 



Figure 1:- Chest CT angiography. (a) axial, (b) axial parenchymal window, (c) coronal and (d) sagittal:

Unencapsulated homogenous fat attenuating region extending from the upper mediastinum to the diaphragm and partially involving the left oblique fissure supporting the diagnosis of mediastinal lipomatosis.

\section{Discussion:-}

Mediastinal lipomatosis is a relatively common benign cause of mediastinal widening [3]. It is the result of an excessive proliferation of mature fat cells. Histologically, lipomatosis is indistinguishable from lipoma except for the lack of circumscription [1].

\section{Contributing factors}

Several contributing factors have been described in literature like obesity, Cushing's syndrome, alcohol abuse, diabetes, prolonged steroid use, congenital malformations, lipomatous abnormalities in the family or other endocrinologic abnormalities [1,2]. The idiopathic origin has also been reported in the literature [2], which was the case of our patient who had presented none of the above-mentioned factors.

\section{Symptoms}

Mediastinal lipomatosis is usually asymptomatic and fortuitously discovered. However, it can become compressive and cause cough, dyspnea even respiratory distress, chest pain, dysphagia, cardiac arrhythmia or superior vena cava syndrome [2-5]. Our patient had acute chest pain with dorsal irradiation. The physical examination can be quite normal, as it can regain dyspnea, obesity and signs associated with Cushing's syndrome [5]. Our patient had no evidence of obesity or steroid excess.

\section{Imaging}

In most cases, mediastinal lipomatosis is fortuitously identified by anterior and superior mediastinal widening on chest X-rays without any deformity of the trachea. In our patient's case, in addition to mediastinal widening, there was an aspect of segmental collapse of the left lower lobe which is relatively rare presentation. The examination of choice is computed tomography or, less often, MRI, it allows not only to confirm the diagnosis of mediastinal lipomatosis but also to define its extent. Adipose tissue can extend from the upper mediastinum to the diaphragm and can involve the heart and lungs [5]. CT is also useful for eliminating a vascular or tissue cause that may require a biopsy. 


\section{Differential diagnosis}

Mediastinal lipomatosis can easily be confused with other causes of mediastinal widening or pulmonary collapse. In case of acute symptomatology, emergencies should be excluded first. These include aortic dissection, esophageal rupture, trauma, haemorrhage or mediastinitis [5]. In our patient's case, left lower lobe segmental lung collapse was suggestive of pulmonary embolism. Outside the emergency, causes of mediastinal widening include lymphoma, lung cancer, thymoma and aneurysms of great vessels [2]. All of these differential diagnoses can be distinguished from benign lipomatosis by imaging, as well as clinical data and electrocardiogram.

\section{Complications}

Although mediastinal lipomatosis is considered benign, it has certain clinical implications. In the case presented by Peek et al, mediastinal lipomatosis was responsible for paralysis of the right hemidiaphragm secondary to phrenic nerve compression [6]. Mediastinal lipomatosis can also cause low voltages on the electrocardiogram [7]. The compression of the superior vena cava, making central venous catheterization difficult, has been cited as a complication [8]. Huang and al., reported a case of primary mediastinal large B-cell lymphoma in mediastinal lipomatosis [9]. A more significant complication is laryngeal compression secondary to excess adipose tissue in the mediastinum leading to airway compromise and right ventricular outflow tract obstruction [10,11].

Treatment

The treatment of mediastinal lipomatosis depends on the degree to which it affects the patient. Mediastinal lipomatosis is usually asymptomatic and no treatment is necessary. In the case the patient is obese, weight loss is strongly recommended [5]. Tapering off steroids is usually needed in steroid induced lipomatosis [3]. However, in case of symptoms related to mass effect, complete surgical resection or surgical reduction may be necessary [12], as in the case presented by Nguyen et al [13]. The clinical episode presented by our patient was transient and required no treatment.

\section{Conclusion:-}

Mediastinal lipomatosis is a benign cause of mediastinal widening on chest radiography. The diagnosis is made on the CT. Medical emergencies and malignancies should be excluded first.

\section{Conflict of interest}

The authors declare that they do not have any conflict of interests.

\section{References:-}

1. Sreekrishna Kanth Donepudi, James C. Greene, Rose Mary S. Stocks. Lipomatosis of the neck: Case report and literature review. International Journal of Pediatric Otorhinolaryngology Extra (2010) 5, 39— 41.

2. Aggarwal D, Mohapatra PR. Mediastinal lipomatosis presenting as right lower lobe lung collapse. Int J Health Sci Res. 2017; 7(4):477-479.

3. Navkiran Kaur,Jasvir Singh, Samrin Haq, Sugandha Garg, and Simmi Bhatnagar. Pleural and Mediastinal Lipomatosis with Subpleural Fat as a Mimicker of Pleural Effusion- A Rare Case Report. J Clin Diagn Res. 2017 Jul; 11(7): TD03-TD04.

4. Camille Taillé, MD; Muriel Fartoukh, MD; Rémi Houël, MD; Hicham Kobeiter, MD; Philippe Rémy, MD; and François Lemaire, MD. Spontaneous Hemomediastinum Complicating Steroid-Induced Mediastinal Lipomatosis. CHEST 2001; 120:311-313.

5. Fonseka, MD, Natasha and Ruel, MD, Ewa (2013) "A Benign Cause of Widened Mediastinum: A Case of Mediastinal Lipomatosis," The Medicine Forum: Vol. 14, Article 8.

6. Peek D, Heijmen R, Ernst S, Schepens M. Extensive mediastinal lipomatosis in a patient with severe aortic valve stenosis. Eur J Cardiothorac Surg 2002 Mar;21(3):564-5.

7. Puttarajappa $\mathrm{C}$, Dhoble A. Mediastinal lipomatosis as a cause of low voltage complexes on electrocardiogram and widened mediastinum: A case report. Cases Journal 2008 Sep;1(1):171.

8. Gombar S, Mitra S, Thapa D, Gombar KK, Pathak R Anesthetic considerations in steroid-induced mediastinal lipomatosis. Anesth Analg. 2004 Mar;98(3):862-4.

9. Huang C, Huang W, Shih J, Yang P. Primary mediastinal large B-cell lymphoma mimicking liposarcoma. Med Oncol 2008 25:284-6.

10. Enzi G. Multiple symmetrical lipomatosis: an updated clinical report Med 1984; 63:56-64. 
11. Bulakci M, Yahyayev A, Ucar A, Erer B, Erer B, Dursun M. Unusual cause of right ventricular outflow tract compression: mediastinal lipomatosis. J Thorac Imaging. 2011 Nov;26(4): W134-6.

12. Antonio D'Andrilli, Camilla Vanni, Federico Venuta, and Erino A. Rendina. Critical tracheal stenosis caused by mediastinal lipomatosis: Long-term efficacy of airway stenting. The Journal of Thoracic and Cardiovascular Surgery. 2015;149: e109-10.

13. Nguyen K, Hoeffel C, et al. Mediastinal Lipomatosis. South Med J vol 91, No. 12. 